Brit. Heart F., 1967, 29, 129.

\title{
Perforation of Peptic Ulcer Complicating Myocardial Infarction
}

\author{
PAUL CASSELL* AND RICHARD NICHOLSON \\ From Department of Thoracic Surgery, Westminster Hospital, London S.W.1, and St. Luke's Hospital, \\ Guildford, Surrey
}

The initiation and activation of peptic ulcer have been recognized for many years to occur in individuals subjected to stress. Attention has been drawn to this phenomenon following burns, fractures, and surgery upon the heart and brain and, indeed, this complication has been referred to as a "bedsore of the gut" (Lancet, 1960).

However, though myocardial infarction is a common condition inducing a state of stress, only rarely has peptic ulceration been reported as occurring during the acute phase of the disease. The purpose of this article is to describe two cases of perforation of an activated peptic ulcer occurring within two days of this event. This particular complication has only been documented twice (Branwood, 1947; Shipp et al., 1959) and is recorded again because it is of practical importance in the differential diagnosis of upper abdominal pain arising after a myocardial infarct.

\section{CASE REPORTS}

Case 1. The patient was a 65 -year-old civil servant who gave a history of angina over the preceding six months but of no dyspeptic symptoms. In March 1964, while walking back from his lunch, he suffered a sudden epigastric pain and a tightness in his chest. During the afternoon the pain spread towards his left shoulder and down the arm. When seen later that day at home he had no abnormal signs other than a pericardial rub, and an electrocardiogram showed an anterior myocardial infarction. He was given sedatives and anticoagulant therapy was started.

Over the next 48 hours his condition improved and then he experienced again severe epigastric pain. This was presumed, reasonably enough in the circumstances, to be an extension of the original lesion, and his sedation was increased. Twenty-four hours later his condition had deteriorated, the pain had persisted, and he had

Received Octcber 4, 1965.

* Present address: Royal Berkshire Hospital, Reading. started copious vomiting of bile-stained fluid. On examination he was cold, ashen, and perspiring, with a pulse rate of 120 and a blood pressure of $120 / 80 \mathrm{~mm} . \mathrm{Hg}$. His abdomen was distended and tympanitic, and bowel sounds were absent. There was a band of resonance above the liver. It was judged that perforation of stomach or bowel had occurred and he was admitted to hospital as an emergency. Plain radiographs confirmed the presence of air in the peritoneal cavity. In view of the grave state of the patient, conservative treatment was begun. He died six hours later.

Necropsy confirmed the diagnosis of a recent myocardial infarction with complete occlusion of the anterior branch of the left coronary artery. The first part of the duodenum was perforated at the site of a chronic ulcer on its anterior wall and there were signs of early peritonitis.

Case 2. The patient, a storekeeper aged 48, was admitted to hospital in March 1964, complaining of a tight constricting retrosternal pain which did not radiate and could not be relieved. He had a previous history of angina for one year treated by his practitioner with glyceryl trinitrate and initially by phenindione (dindevan). Following this he passed melæna stools for which he was admitted to another hospital and received medical treatment. He had suffered from post-prandial epigastric discomfort and heartburn on and off since his youth. When examined it was noted that he was not in failure. The blood pressure was $180 / 110 \mathrm{~mm}$. $\mathrm{Hg}$ and his pulse was 100 , regular, and of good volume. The heart and lungs were clinically normal as was the abdomen apart from a liver palpable two finger breadths below the costal margin. An electrocardiogram confirmed a diagnosis of myocardial infarction and treatment with complete bed-rest and morphine was begun.

Ten hours later his blood pressure fell initially to $120 / 80 \mathrm{~mm}$. $\mathrm{Hg}$ and then gradually to $90 / 70 \mathrm{~mm}$. $\mathrm{Hg}$. In view of the grave condition of the patient it was considered justifiable, despite the previous history of peptic ulceration, to give anticoagulants. Accordingly heparin therapy was commenced and a metaraminol (aramine) 129 
On the next day the patient complained of severe abdominal pain. He was shocked, with a systolic blood pressure of $100 \mathrm{~mm} . \mathrm{Hg}$ and a pulse rate of 90 , and the gastric aspirate was heavily blood-stained and "coffeeground" in appearance. The heparin was reversed, morphine was given, and the pain settled, only to return in the left hypochondrium eight hours later, on this occasion however being accompanied by rigidity and a silent abdomen. Plain abdominal radiographs showed air and fluid in the peritoneal cavity, but because his blood pressure had fallen still further, despite metaraminol, conservative management of his perforation was decided upon. Once more the patient recovered and progressed satisfactorily for five days and then a gastrografin was done which showed a perigastric abscess on the greater curvature at the pyloric antrum and a deformed duodenal cap. Shortly after this further hæmatemesis and melæna occurred requiring a transfusion of four pints of blood and again five days later a third hæmatemesis demanded a further blood transfusion of six pints. Following this gradual progress was made, and eventually after two months he was discharged home. He is now well, with no gastro-duodenal symptoms, and is back at work. A recent barium meal has demonstrated a pyloric ulcer with extensive deformation of the pylorus and duodenum.

\section{Discussion}

The case histories of two patients who perforated peptic ulcers during the acute phase of myocardial infarction are reported. The frequent association of coronary artery disease with chronic peptic ulcer has been noted in several series (Junet, 1944; Hochrein and Schleicher, 1941). Further, an increased incidence of peptic ulceration has been found at necropsy in patients dying from myocardial infarction (Morrison and Gonzalez, 1952).

The adverse effect of cortisone therapy on peptic ulcers is well known, and Gray and his colleagues have demonstrated that raised blood adrenocortical hormone levels have been accompanied by increased gastric secretory activity (Gray et al., 1951; Gray and Ramsey, 1957). Such a rise has been shown to occur immediately after myocardial infarction.

It is surprising, therefore, that few records have been published of perforation occurring during the management of myocardial infarction. It is possible that this association is not as rare as the lack of reports implies, because unless a necropsy is done, the signs and symptoms produced by a perforation might reasonably be attributed to an extension of the infarction. If the possibility of this complication is borne in mind, prompt treatment may avert the otherwise fatal outcome.

A patient presenting in a state of shock and complaining of severe epigastric pain always produces a problem in differential diagnosis, perforated peptic ulcer and myocardial infarction being two of the most common conditions to be considered. Indeed on some occasions it is not possible to differentiate between them on clinical grounds alone and ancillary investigations have to be utilized in order to establish the correct diagnosis and initiate the appropriate treatment. In the patient with a proven myocardial infarction it is not unusual for further exacerbations of pain to occur, due to extension of the original lesion. It is in circumstances like these when recrudescence of epigastric pain is attributed to this cause that perforation of an acute or activated chronic peptic ulcer may be overlooked.

This particular difficulty is well demonstrated by our two patients. The first had no history of dyspepsia and the correct diagnosis of his second attack of pain was not made for 24 hours, until the onset of peritonitis with abdominal distension and ileus. On the other hand, the second patient had suffered from indigestion for years and had passed a melæna stool a few months previously. In addition, the perforation was preceded by a hæmatemesis which gave warning of the activation of his peptic ulcer.

The occasional occurrence of paralytic ileus in the shocked infarction patient may render the task of excluding a perforation even more difficult. In this event the detection of subdiaphragmatic air by percussion and radiological examination is a reliable method of differentiation.

The prognosis of a patient suffering from these two potentially fatal lesions might be expected to be bad. It is encouraging to note that two of Shipp's four patients survived under conservative management, as did one of ours. Branwood's case survived six days and then died from left ventricular failure. Obviously if an early diagnosis is made and strenuous supportive measures instituted a successful outcome is not precluded in some patients.

Although gastroduodenal ulceration and its sequelæ complicate myocardial infarction infrequently, it is nevertheless advisable to institute more energetic prophylactic measures than the avoidance of anticoagulant therapy. It is suggested that it is important to endeavour to maintain an adequate oral intake throughout the period of stress. Unfortunately, it is at this time that the medical attendants are most preoccupied with the immediate problem of the infarction and its sequelæ. The patient himself at this time has no inclination to eat or drink, if he is not actually vomiting.

In addition to this, positive findings to a careful inquiry about a patient's dyspeptic history, which may be forthcoming only as a reluctant admission 
to the "odd spot of wind", seem to demand a full "ulcer regime". Above all a careful watch should be kept for signs of hæmorrhage or perforation, and further pain should not be automatically attributed to the cardiac lesion until activation of a peptic ulcer has been excluded.

\section{SUMMARY}

Two cases of perforated peptic ulcer complicating the early management of myocardial infarction are recorded.

The difficulties encountered in diagnosis and treatment are discussed and the principles of prophylaxis outlined.

\section{REFERENCES}

Branwood, A. W. (1947). A case of perforated duodenal ulcer and cardiac infarction. Brit. Heart $\mathcal{F}$., 9, 263.
Gray, S. J., Benson, J. A., Reifenstein, R. W., and Spiro, H. M. (1951). Chronic stress and peptic ulcer. I. Effect of corticotrophin (ACTH) and cortisone on gastric secretion. F. Amer. med. Ass., 147, 1529.

- , and Ramsey, C. G. (1957). Adrenal influences upon the stomach and the gastric responses to stress. Recent Progr. Hormone Res., 13, 583.

Hochrein, M., and Schleicher, I. (1941). Ulcus pepticum und Angina pectoris. Münch. med. Wschr., 88, 328.

Junet, R. (1944). Ulcères gastro-duodénaux et syndromes coronariens. Helv. med. Acta, 11, 75.

Lancet (1960). Bedsores of the gut. 1, 1393.

Morrison, L. M., and Gonzalez, W. F. (1952). The relationship of chronic peptic ulcer to coronary thrombosis. Amer. F. med. Sci., 224, 314.

Shipp, J. C., Sidel, V. W., Donaldson, R. M., and Gray, S. J. (1959). Serious complications of peptic ulcer after acute myocardial infarction. New Engl. f. Med., 261, 222. 